\section{Play it again, Mr Sandman}

In a scene familiar to all, a fractious child is sent to bed in the belief that the sleep that will surely follow will allow the child to get some much needed 'rest'. But the idea that sleep is simply a rest period has been repeatedly challenged over the years. Initially, the challenge was provided by the electrophysiological descriptions of the sleep-wake cycle. More recent findings have linked sleep and the hippocampus with the consolidation of neuronal temporal codes for spatial memory. However, we still know little about sleep. Two recent papers in Science provide thoughtprovoking additions to this literature.

In the first paper, Robert Stickgold and colleagues focused on the effect of extended mental activity on hypnagogic mentation - the replay of mental imagery at the onset of sleep. The experiment was designed to evaluate the source and the function of hypnagogic images. The participants played the computer game Tetris ${ }^{\circledR}$ for extended periods over three days and were asked to report mentation during the first hour of attempted sleep each evening. Three groups participated in the experiment: novices, experts and amnesic patients with bilateral medial temporal lobe damage. All subjects reported intrusive stereotypical images of the blocks used in the game at sleep onset. This was true even for the amnesic patients who could not recall playing the game. One interpretation of this result is that hypnagogic images do not require intact declarative memory systems. Another intriguing aspect of this study is that although playing Tetris might be expected to involve procedural learning (which is intact in amnesic subjects), the amnesic patients in this study did not show the same level of improvement as the novice players. Further work will be required to clarify this area, but it does seem that the source of hypnagogic images and the improved performance may rely on different areas of the brain.

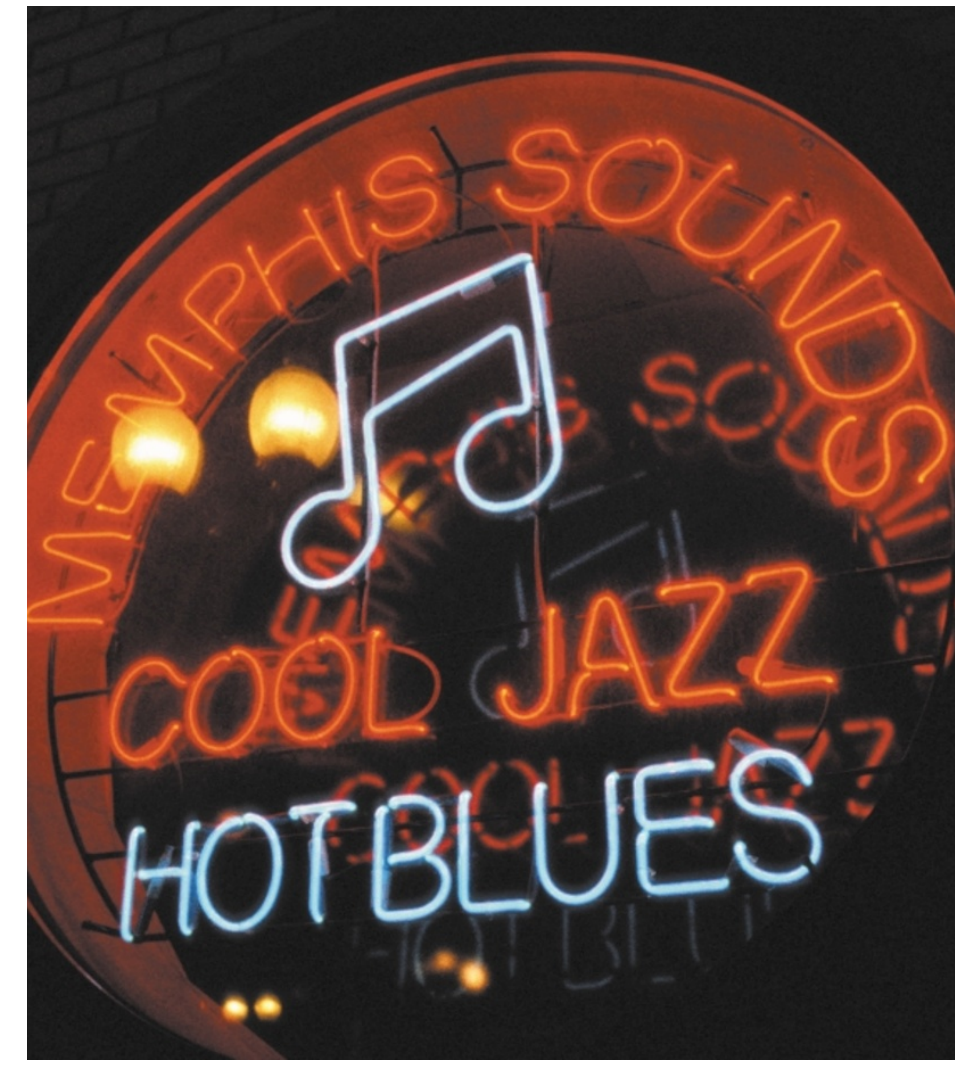

The second study provides evidence for the role of sleep in birdsong learning. Dave and Margoliash recorded the activity of single neurons in the forebrain nucleus robustus archistriatalis (RA) of zebra finches. This area is analogous to the motor cortex in mammals and its descending projections represent the output of the forebrain song system. Recordings from RA in individual birds were made while each bird was singing and again during playback of the bird's song while it was asleep. The timing and structure of the neural activity elicited by the playback of song during sleep matched the activity recorded during singing. Activity during singing leads the sounds that are produced, thus the matching auditory responses represent a prediction of the following sound based on the sequence of the preceding sounds. Such predictions may lie at the heart of the computations involved in vocal learning. Moreover, the spontaneous activity of the RA neurons during sleep matched their sensorimotor activity, and thereby may provide a form of song replay for motor learning.

In conclusion, these two studies indicate that the replay of sensory experience during sleep may have complex effects on different forms of learning and memory. So although it may be some time before the secrets of the Sandman are finally revealed, these two studies demonstrate that we are now able to tackle this problem with a wide range of techniques.

Peter Collins

\section{(9) References and links} ORIGINAL RESEARCH PAPERS Stickgold, R. et al. Replaying the game: hypnagogic images in normals and amnesics. Science 290, 350-353 (2000) | Dave, A. S. \& Margoliash, D. Song replay during sleep and computational rules for sensorimotor vocal learning. Science $\mathbf{2 9 0}$ 812-816 (2000)

FURTHER READING Brainard, M. S. \& Doupe A. J. Auditory feedback in learning and maintenance of vocal behaviour. Nature Rev. Neurosci. 1, 31-40 (2000)

ENCYCLOPEDIA OF LIFE SCIENCES Amnesia WEB SITE Daniel Margoliash

\section{IN THE NEWS}

Purple haze

A recent flurry of reports in the UK media focused on a paper in Nature Neuroscience on the addictive properties of the psychoactive component of marijuana. They concerned a report by Steven Goldberg that self-administration behaviour is maintained by delta-9-tetrahydrocannabinol (THC) in squirrel monkeys. Previous studies that have tried to establish reliable selfadministration behaviour in animals with $\mathrm{THC}$ have been unsuccessful, indicating that marijuana might have less potential for abuse than other drugs such as heroin and cocaine. The press interest was stimulated by the likely impact of these findings on the polarized debate in the UK media regarding the issue of whether to decriminalize marijuana. So how did the media present the story?

Marijuana "is as addictive as heroin"

A study into the addictiveness of cannabis has concluded that the drug can be as compulsive as cocaine or heroin. The

findings will come as a blow to those campaigning for the legalisation of marijuana. The research by Steven Goldberg... used a standard test for addictiveness in laboratory animals and found little difference between the effects of cocaine, heroin and cannabis. The Independent

A detail missing from the piece was that self-administration of THC was obtained in monkeys that had previously been trained to self-administer cocaine - a standard test of the addictive properties of psychoactive drugs. Future research will be required to establish whether drug naïve monkeys will also selfadminister marijuana at the low doses used in this study. And this may be important in the final analysis of the abuse potential of marijuana.

Peter Collins 